\title{
Serum B-type natriuretic peptide levels as a marker for anthracycline-induced cardiotoxicity
}

\author{
YA-DI WANG ${ }^{1,2}$, SU-XIAN CHEN ${ }^{3}$ and LI-QUN REN ${ }^{1}$ \\ ${ }^{1}$ Department of Experimental Pharmacology and Toxicology, School of Pharmacy, Jilin University, Changchun, Jilin 130021; \\ ${ }^{2}$ Department of Oncology, Liaoning Medical University, Jinzhou, Liaoning 121001; ${ }^{3}$ Department of Pathology, \\ The Third Affiliated Hospital of Liaoning Medical University, Jinzhou, Liaoning 121000, P.R. China
}

Received February 4, 2015; Accepted March 15, 2016

DOI: $10.3892 / \mathrm{ol} .2016 .4424$

\begin{abstract}
Observational and experimental studies have produced inconsistent evidence about the association of serum levels of B-type natriuretic peptide (BNP) with anthracycline-induced cardiotoxicity (AIC). Therefore, the current meta-analysis examined the association between serum BNP levels and AIC by using data from high quality studies published in peer-reviewed journals. Relevant studies were identified through literature searches of China National Knowledge Infrastructure (CNKI), Web of Science, PubMed, Google Scolar and China BioMedicine (CBM). STATA software was used in this meta-analysis for statistical analysis. In addition, the crude standardized mean difference (SMD) with 95\% confidence interval (CI) for the highest vs. the lowest category of serum BNP levels was calculated. A total of 8 independent case-control studies, containing 126 AIC patients and 569 healthy controls, were included for the current meta-analysis. The results indicated a significant difference in serum BNP levels between the cardiotoxic group and normal group, with respect to post-treatment and pretreatment with anthracyclines. Specifically, the serum levels of BNP increased remarkably after treatment with anthracyclines in the cardiotoxic group, compared with the normal group. No publication bias was detected in this meta-analysis. The findings of the present study provide strong evidence that serum BNP levels may be associated with AIC.
\end{abstract}

\section{Introduction}

Anthracycline agents are effective drugs utilized for a variety of solid and haematological malignancies $(1,2)$. Anthracyclines

Correspondence to: Dr Li-Qun Ren, Department of Experimental Pharmacology and Toxicology, School of Pharmacy, Jilin University, 1266 Fujin Road, Changchun, Jilin 130021, P.R. China

E-mail: renliqun1027@163.com

Key words: serum B-type natriuretic peptide levels, anthracycline-induced cardiotoxicity, post-treatment, pretreatment, standardized mean difference, meta-analysis, case and control study, confidence interval significantly improve the overall 5 -year survival $(>80 \%)$ in pediatric cancers $(3,4)$. Nevertheless, anthracyclines are capable of inducing short- and long-term cardiotoxicity $(2,5)$. In the clinic, anthracycline-induced cardiotoxicity (AIC) is characterized as ventricular dysfunction with no symptoms in $557 \%$ of patients, and clinical heart failure after cardiomyopathy in $\leq 16 \%$ of the patients $(3,6,7)$. In general, the risk of developing AIC is closely linked to the cumulative dose of anthracyclines $(8,9)$. Furthermore, other factors such as age and gender may also contribute to the occurrence of AIC (1). Once patients exhibit symptoms of cardiac failure, reversal of the condition via termination of anthracycline therapy may become impossible (10). Thus, it is important to detect AIC as early as possible (9). Multiple studies have demonstrated that B-type natriuretic peptide (BNP), a neurohormone secreted in the cardiac ventricles, reflect left ventricular (LV) end diastolic pressure and pulmonary capillary wedge pressure, and may independently predict AIC $(1,11)$.

BNP, originally observed in extracts of porcine brain, belongs to the family of natriuretic peptides (NPs), and possesses the ability to promote diuresis, natriuresis, vasodilatation and repress the renin-angiotensin-aldosterone system (RAAS) (12). BNP regulates homeostasis of water and salt excretion, and maintains blood pressure largely through suppressing the RAA axis and damaging the cardiac sympathetic nerve activity (13). Measurement of NPs has been widely utilized for the detection and monitoring of patients with systolic dysfunction $(14,15)$. As an important member of NPs, BNP is found up-regulated in a number of cardiac pathologies related to hemodynamic overload and elevated cardiac fibrosis (16-18). In addition, serum BNP levels are increased in patients with symptomatic LV dysfunction, indicating that BNP may be linked to the prognosis of heart failure (19). In recent years, serum BNP level was demonstrated to have relevance to the pathogenesis of $\mathrm{AIC}(9,11)$. BNP is secreted by ventricular cardiac cells and released into the blood in response to myocardial stretch, ventricular dilatation and increased pressure load of LV chambers, which are influenced by LV diastolic dysfunction (20-22). Elevated synthesis of BNP may be stimulated by myocardial ischemia, LV wall tension and pressure overload, all of which could reflect alterations in ventricular function in heart failure patients (23-25). On the other hand, anthracyclines cause degeneration of ventricular 
muscles and consequently exhibit characteristics of congestive heart failure (26). More notably, anthracyclines can induce myocardial ischemia and increase pressure load (8). From these aspects, it is rational to postulate that serum BNP levels may be associated with AIC. Previous studies investigated the impact of serum BNP levels on the detection and prognosis of AIC, but their findings are contradictory $(8,25)$, the present study endeavored to use a meta-analysis approach to examine the clinical value of serum BNP levels on the prediction and management of AIC.

\section{Materials and methods}

Search strategy. Relevant studies were identified by searching the medical literature databases China National Knowledge Infrastructure (CNKI; available from www.cnki.net), Web of Science (available from webofknowledge.com), PubMed (available from www.ncbi.nlm.nih.gov/pubmed), Google Scolar (available from scholar.google.com and China BioMedicine (CBM; available from www.sinomed.ac.cn) databases. The following key terms were used for the systematic search: ('Anthracyclines' or 'Anthracycline cardiotoxicity' or 'Anthracycline' or 'Anthracycline antibiotics' or 'Anthracycline-induced cardiotoxicity' or 'Anthracycline-Induced Cardiomyopathy' or 'anthracycline-induced cardiac toxicity') and ('Natriuretic Peptide, Brain' or 'BNP 32' or 'Nesiritide' or 'Type B Natriuretic Peptide' or 'B Type Natriuretic Peptide' or 'BNP' or 'B-Type Natriuretic Peptide' or 'Brain Natriuretic Peptide 32' or 'Natrecor' or 'pro-brain natriuretic peptide (1-76)' or 'N-terminal pro-BNP' or 'proBNP (1-76)' or 'NTproBNP' or 'NT-BNP' or 'Amino-terminal pro-brain natriuretic peptide' or 'NT-pro-BNP' or 'aminoterminal pro-B-type natriuretic peptide' or 'pro-B-type natriuretic peptide' or 'N-Terminal Pro-B-Type Natriuretic Peptide' or 'Brain Natriuretic Peptide'). No language restrictions were imposed in the current meta-analysis. Other potentially relevant studies were also identified by a manual search of the reference list of original reports.

Selection criteria. The following criteria were used to select published articles in our present meta-analysis: i) Human-associated case-control studies focused on the relationship between the serum levels of BNP and AIC; ii) sufficient information on the serum levels of BNP and sample number must be supplied; iii) the article must be published with full text. By contrast, published articles were excluded based upon at least one of the following reasons: i) Articles did not satisfy the inclusion criteria of the current study; ii) studies were letters, reviews and meta-analyses; iii) articles were duplication publications or had overlapping data. After careful reexamination with the search strategy and inclusion/exclusion criteria, only the most recent or complete studies were included in the current meta-analysis.

Data extraction and quality assessment. In order to enhance the credibility and reduce bias, the following relevant data were extracted from each included study: i) Baseline demographics: Year of publication, surname and initials of the first author, country and ethnicity of publication; ii) Participants: Number of cases and controls, sample size, gender and age of subjects and iii) Detection method of serum BNP levels.
The Newcastle-Ottawa Scale (NOS), was used as the criteria for assessment of study quality (27). The NOS criteria were scored based on three perspectives: Subject selection (0-4); comparability of subject (0-2) and clinical outcome (0-3). Total NOS scores ranged from 0 (lowest) to 9 (highest). The included studies were viewed as high quality when score was $\geq 7$. Additional reassessment was performed by authors to resolve discrepancies on NOS scores.

Statistical analysis. The STATA statistical software (Version 12.0, Stata Corporation, College Station, TX, USA) was used for all analyses in this meta-analysis. The association between the serum levels of BNP and AIC was estimated by summary standardized mean difference (SMD) with 95\% confidence intervals (CIs). Statistical significance of pooled SMDs was estimated by the $\mathrm{Z}$ test, the heterogeneity among studies was quantified by Cochran's $Q$-statistic and $I^{2}$ tests (28). If the $Q$-test showed $\mathrm{P}<0.05$ or $I^{2}$ test exhibited $>50 \%$, indicating significant heterogeneity, random effects model was applied, otherwise fixed-effects model was conducted in case of no statistical heterogeneity $(29,30)$. In addition, subgroup analyses were performed on the basis of ethnicity and method to explore potential explanatory variables for the association between the serum levels of BNP and AIC. Sensitivity analysis was conducted to assess the stability of the present study. To reveal possible publication bias, the funnel plot and Egger's linear regression test were performed $(31,32)$. All tests were two-sided and a P-value $<0.05$ was considered to indicate a statistically significant difference.

\section{Results}

Description and characteristics of included studies. Figure 1 presents the study selection process and the reasons for exclusion. Initially, a combination of electronic database search and manual search resulted in 175 articles being retrieved. At the screening stage, two articles were excluded due to duplication and 133 articles were excluded because of the article types (letters, reviews and meta-analyses), if they were not human studies and if they were not related to research topics. Subsequently, another 29 articles were excluded because they were not case-control or cohort studies, or not relevant to serum level of BNP and anthracyclines or not relevant to cardiac toxicity. Finally, three articles with incomplete data were excluded. The remaining 8 articles met all the inclusion criteria and the studies were, published between 2001 and 2014 , with moderate-high study quality $(1,8-11,33-35)$. Table I shows the basic characteristics of included studies on the BNP serum level. The eight cohort studies contained 126 AIC patients and 569 normal participants, with sample size varying from 5-312 participants. Five studies were performed in Asian individuals with two in China, one each in Korea, Thailand and Japan. Three studies were performed in the Caucasian population, with one each in Denmark, Italy and USA. Two studies (Skovgaard D; Cao S) lacked gender information and three studies (Skovgaard D, Lee HS, Aggarwal S) lacked age information. The detection methods included enzyme multiplied immunoassay technique (EMIT), chemiluminescence technology (CIT), indirect immunofluorescence (IFT), fluorescence immunoassay analysis (FIA), chemiluminescence 


\section{\begin{tabular}{|l|l|l|}
\hline Identification & Screening & Included
\end{tabular}}

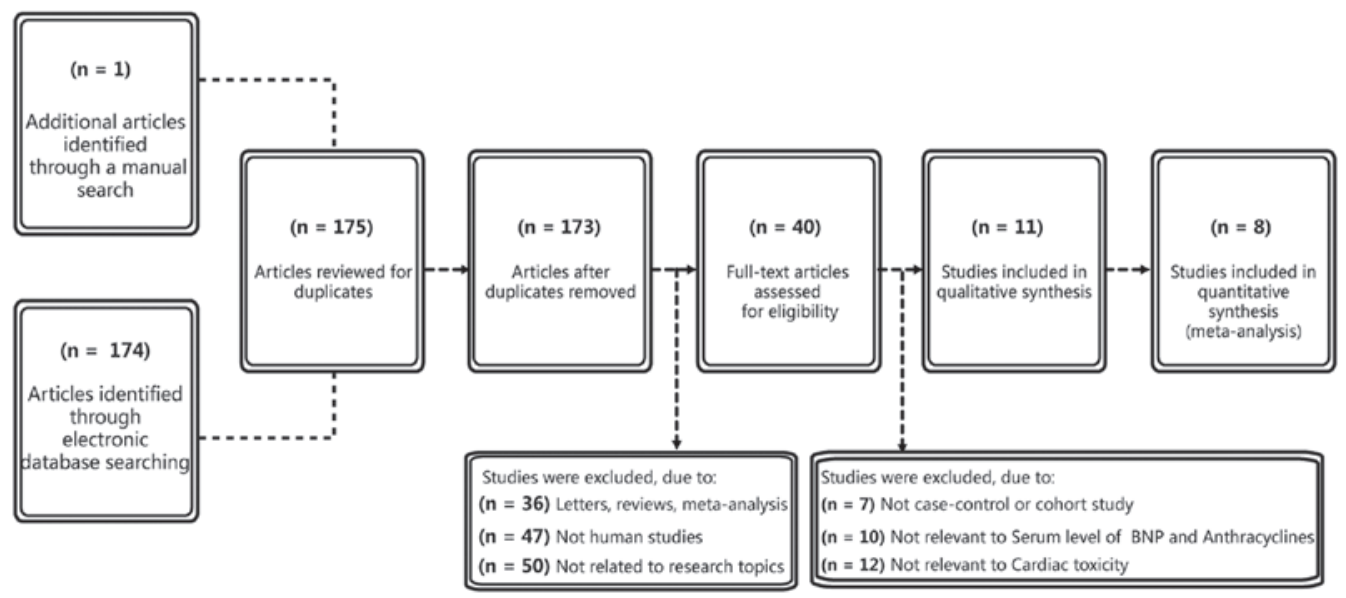

Figure 1. Flow chart showing study selection procedure. Seventeen case-control studies were included in the B-type natriuretic peptide meta-analysis.

immunoassay (CLIA) and radioimmunoassay (RIA). The follow-up time was 3, 4, 18, 48 and 96 weeks. The serum BNP level of cardiotoxic patients and normal participants were expressed as the mean \pm standard deviation.

Quantitative data synthesis. The main result of the present meta-analysis on the relationship between post-treatment serum BNP levels and AIC in cardiotoxic group and normal group was based on random-effects analysis due to $I^{2}>50 \%$ and $\mathrm{P}<0.001$. Significant differences in serum BNP levels in cardiotoxic group and normal group between post-treatment and pretreatment of anthracyclines were detected (cardiotoxic group: $\mathrm{SMD}=0.72,95 \% \mathrm{CI}=0.40-1.03, \mathrm{P}<0.001$; normal group: $\mathrm{SMD}=0.74,95 \% \mathrm{CI}=0.52-0.96, \mathrm{P}<0.001)$. Serum levels of BNP after anthracycline treatment increased remarkably in cardiotoxic group compared with the normal group ( $\mathrm{SMD}=2.50,95 \% \mathrm{CI}=1.11-3.89, \mathrm{P}<0.001)$ (Fig. 2). In addition, subgroup analysis based on ethnicity and detection methods were performed to explore the potential influential factors on the relationship between serum levels of BNP and AIC. Ethnicity stratified analysis showed significantly higher BNP serum levels in post-treatment anthracyclines patients compared with the pretreatment patients in the cardiotoxic group in the Asian, but not in the Caucasian, population (Asian: $\mathrm{SMD}=1.05,95 \% \mathrm{CI}=0.61-1.49, \mathrm{P}<0.001$; Caucasian: $\mathrm{SMD}=0.37,95 \% \mathrm{CI}=-0.08-0.81, \mathrm{P}=0.110)$. Significantly higher BNP serum levels after anthracyclines treatment were observed compared to pretreatment with anthracyclines in both Asian and Caucasian patients (all P>0.05). The serum level of BNP after anthracyclines treatment was notably higher in the cardiotoxic group than the normal group. As for stratified analysis on the basis of detection method, higher BNP serum level was found after anthracyclines treatment in cardiotoxic group with ECIA, IFT method $(\mathrm{P}>0.05)$ but not with FIA method (SMD=0.37, 95\% CI=-0.08-0.81, $\mathrm{P}=0.110)$. While higher BNP serum levels were observed after anthracyclines treatment in the normal group with ECIA, IFT and FIA methods (all $\mathrm{P}<0.05$ ). Post-treatment serum level of BNP was notably higher in the cardiotoxic group compared with the normal group with EMIT, ECIA, CIT, IFT, FIA, RIA (all $\mathrm{P}<0.05)$ but not with CLIA (SMD=8.03, 95\% CI=-6.78-22.84, $\mathrm{P}=0.288$ ) (Fig. 3).

Sensitivity analysis and publication bias. Sensitivity analysis was conducted to determine the influence of one single study on the whole result of the analysis, which could indicate the stability of the study. It demonstrated that no single study affected the pooled SMDs in the meta-analysis of anthracyclines treatment and cardio toxicity (Fig. 4). Finally, the Egger's regression test was applied to detect the publication bias. The result showed symmetrical distributions in the funnel-plot, indicating publication bias in the systematic reviews (Cardiotoxic: Egger's test: $\mathrm{t}=8.28, \mathrm{P}<0.001$; Normal: Egger's test: $\mathrm{t}=5.93, \mathrm{P}=0.002$; Cardiotoxic vs. Normal: Egger's test: $\mathrm{t}=2.40, \mathrm{P}=0.037$ ) (Fig. 5).

\section{Discussion}

The present meta-analysis was performed to evaluate the adverse effects of anthracyclines in cancer patients and to determine the relationship between serum BNP levels and cardiotoxicity. The findings demonstrated that patients with cardiotoxicity displayed increased serum BNP levels after anthracycline treatment compared to pretreatment, the normal people treated by anthracyclines also have higher serum levels of BNP, and patients with AIC have much higher BNP levels than normal individuals. Anthracyclines have the adverse effects of causing cardiotoxic events in cancer patients and BNP responds to end-diastolic pressure. Slight increases in BNP serum levels identify a risk of cardiac dysfunction, suggesting that increased BNP levels can be a diagnostic marker for the risk of cardiotoxicity and even for improving the diagnostic performance of cardiac disease induced by anthracycline-like chemotherapy (11). Low dose of anthracyclines contribute to deterioration of cardiac function in patients with acute lymphoblastic leukemia, and is readily detected by the cardiac biomarker BNP (36). AIC has a 


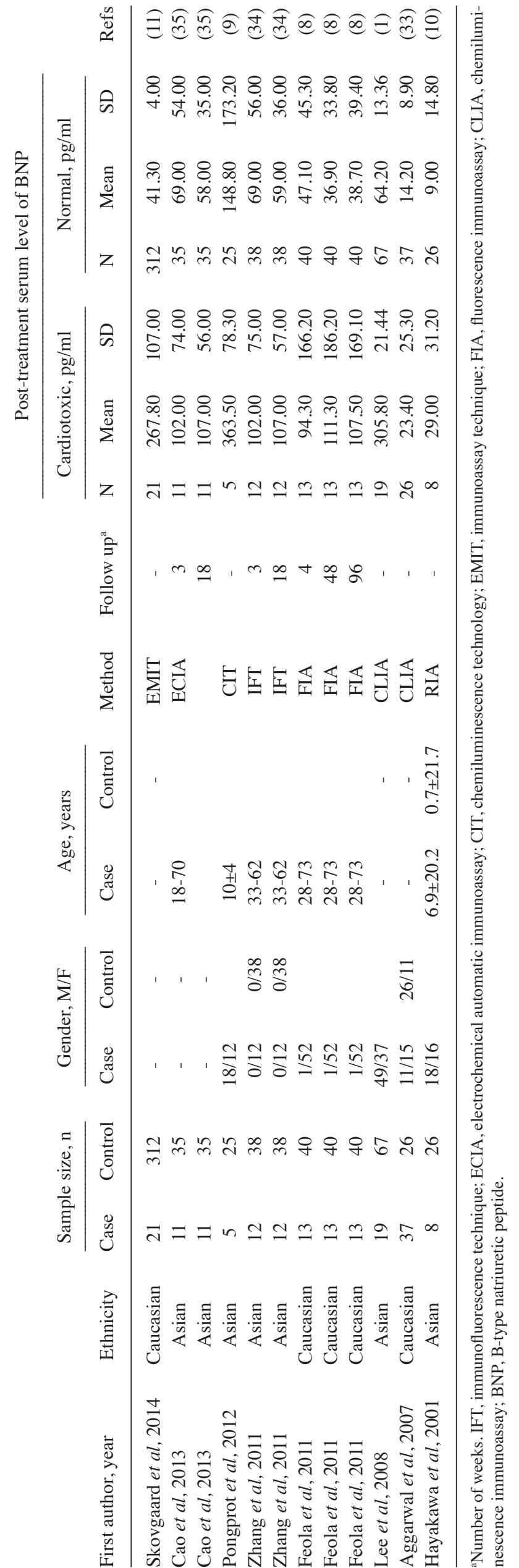



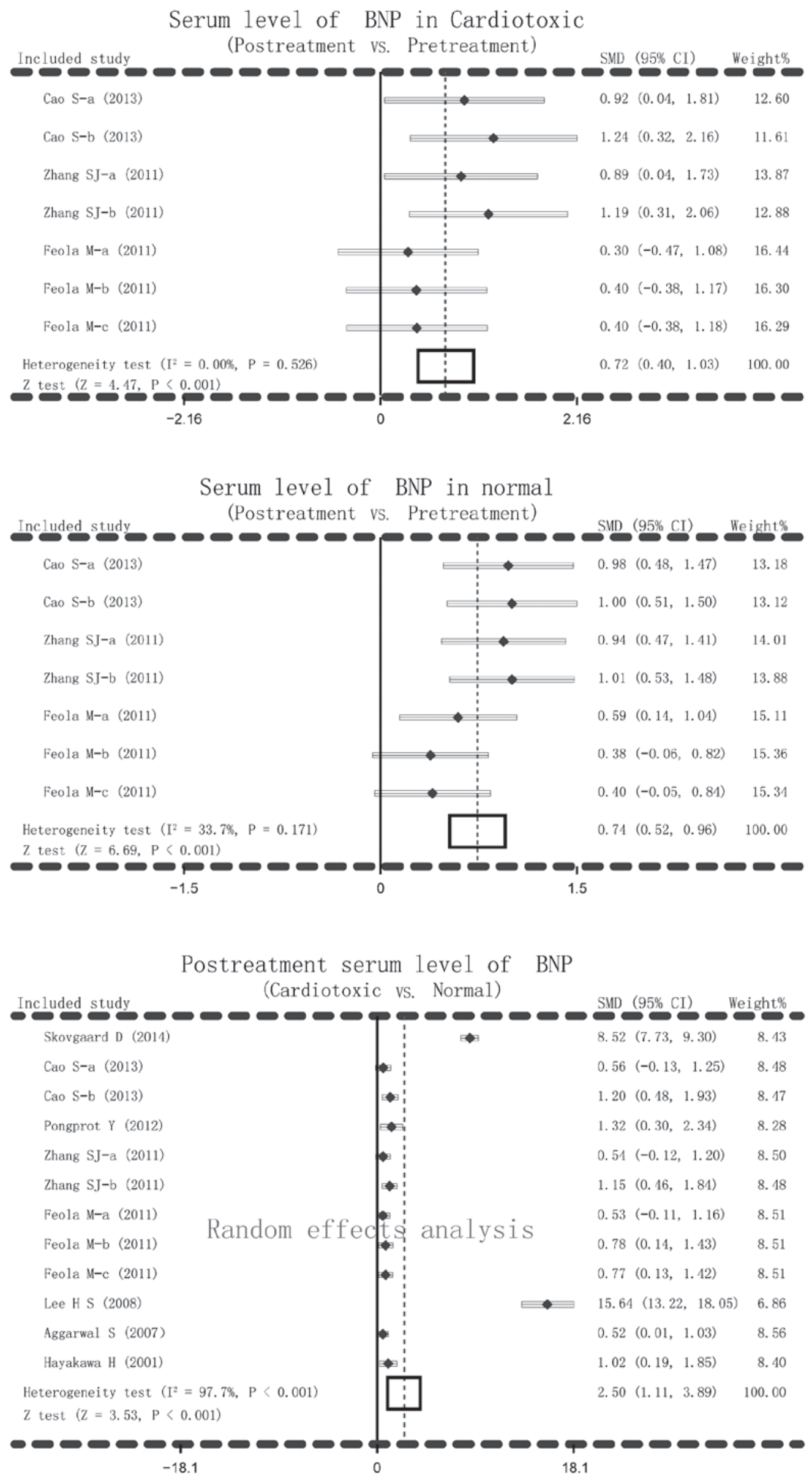

Figure 2. Forest plots for current meta-analysis investigating the association between serum B-type natriuretic peptide levels and anthracycline-induced cardiotoxicity. 
Serum level of BNP in Cardiotoxic

(Ethnicity: Postreatment vs. Pretreatment)

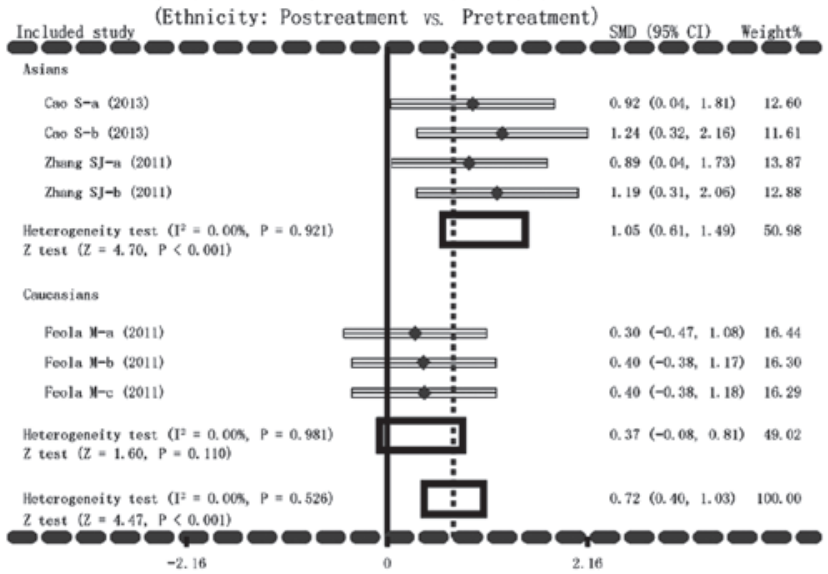

Serum level of BNP in normal

(Ethnicity: Postreatment vs. Pretreatment)

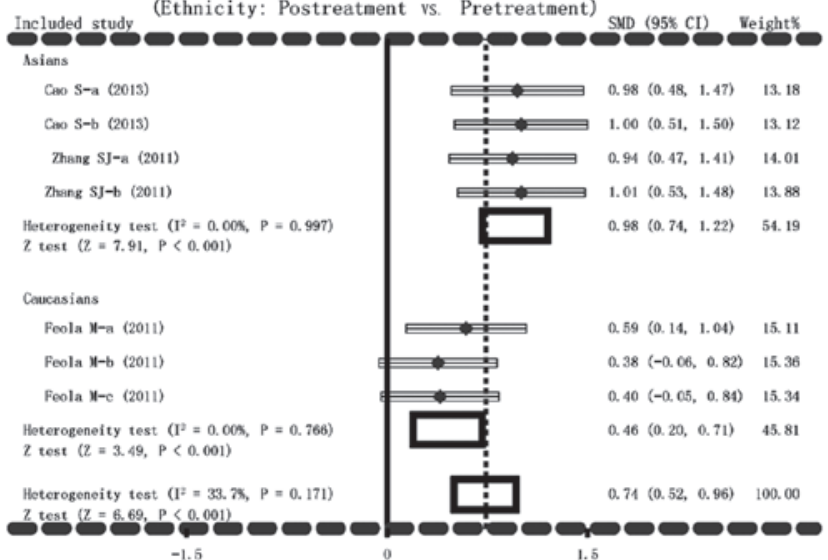

Postreatment serum level of BNP

(Ethnicity: Cardiotoxic vs, Nornal)

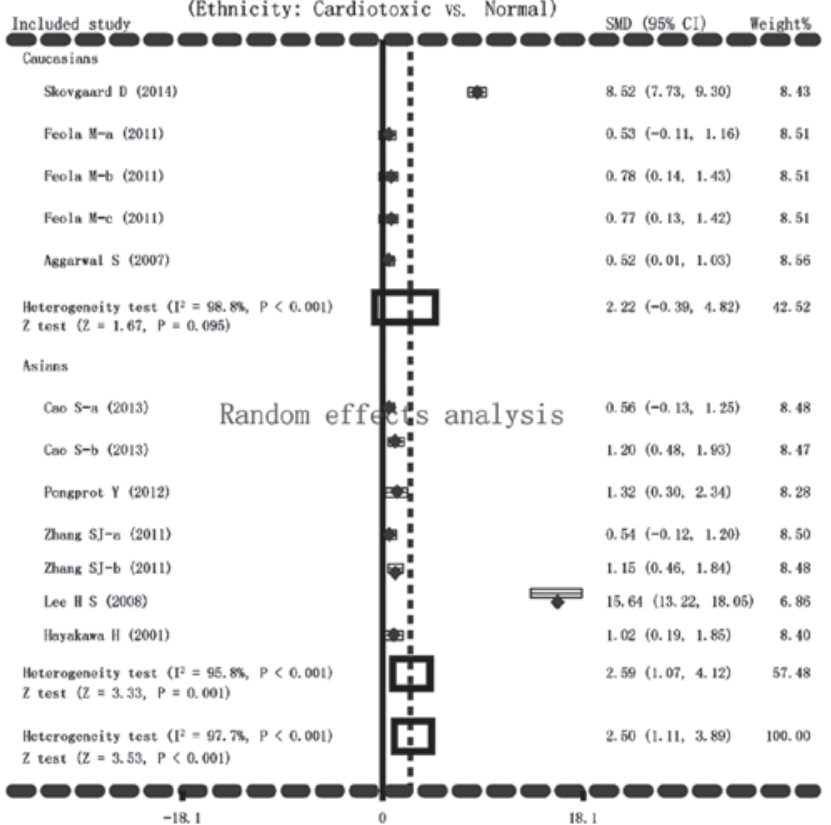

Serum level of BNP in Cardiotoxic

(Method: Postreatment vs. Pretreatnent)

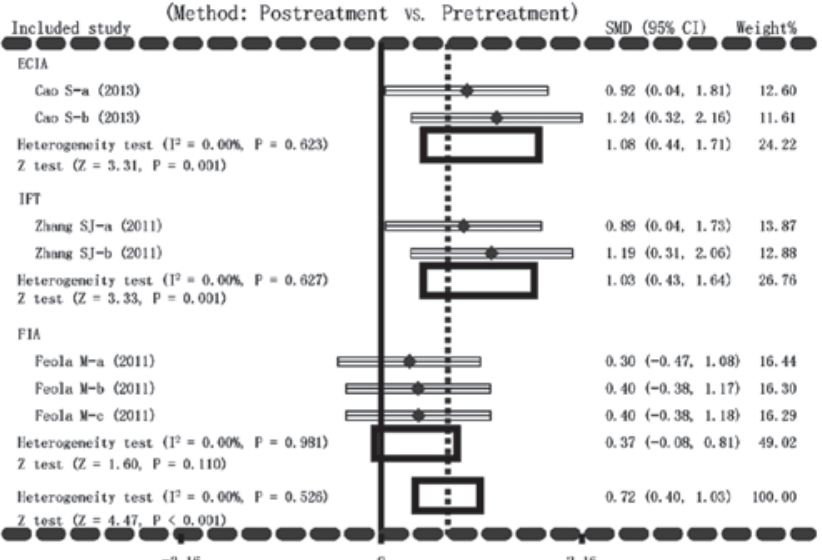

Serum level of BNP in normal

(Method: Postreatment vs. Pretreatment)

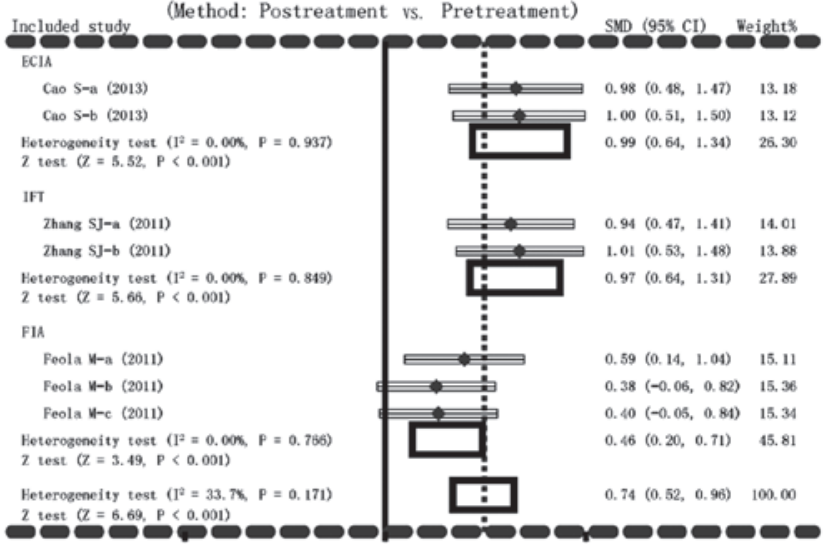

Postreatment serum level of BNP

(Method: Cardiotoxic vs. Normal)

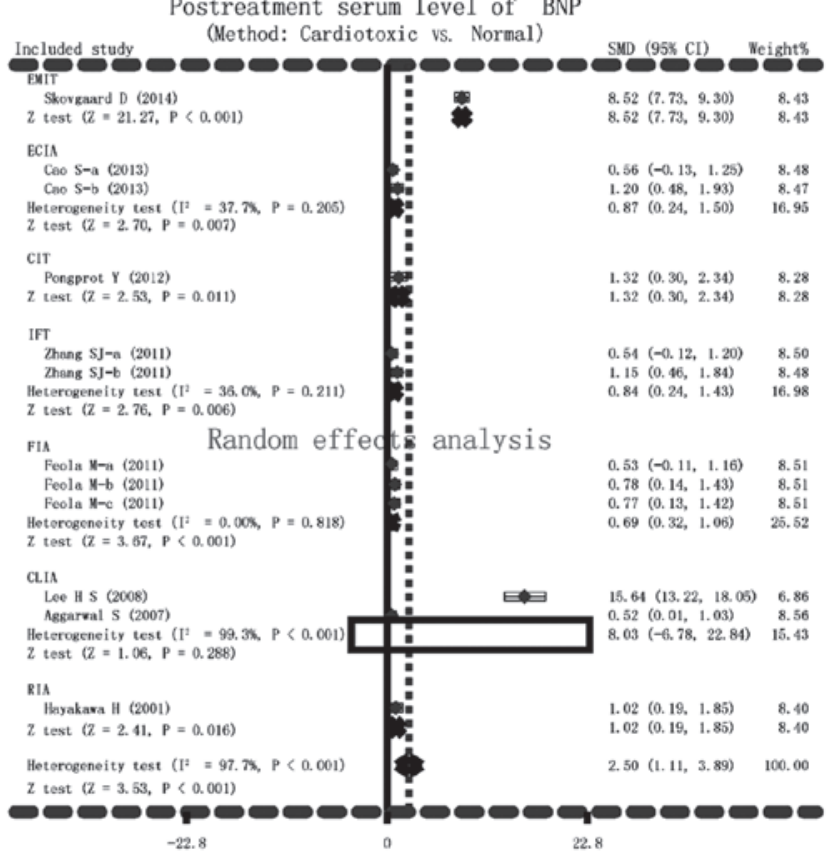

Figure 3. Subgroup analyses by ethnicity and detection methods in current meta-analysis investigating the association between serum B-type natriuretic peptide levels and anthracycline-induced cardiotoxicity.

vital impact on cardiac failure, and elevated BNP serum levels in cardiotoxic patients signify compensatory mechanisms to alleviate the severity of myocardial damage, thus BNP could serve as a useful factor in indicating cardiotoxicity incidence (37). On account of the above working mechanisms of $\mathrm{BNP}$, the meta-analysis results conclude that up-regulated BNP 
Serum level of BNP in Cardiotoxic (Postreatment vs. Pretreatment)

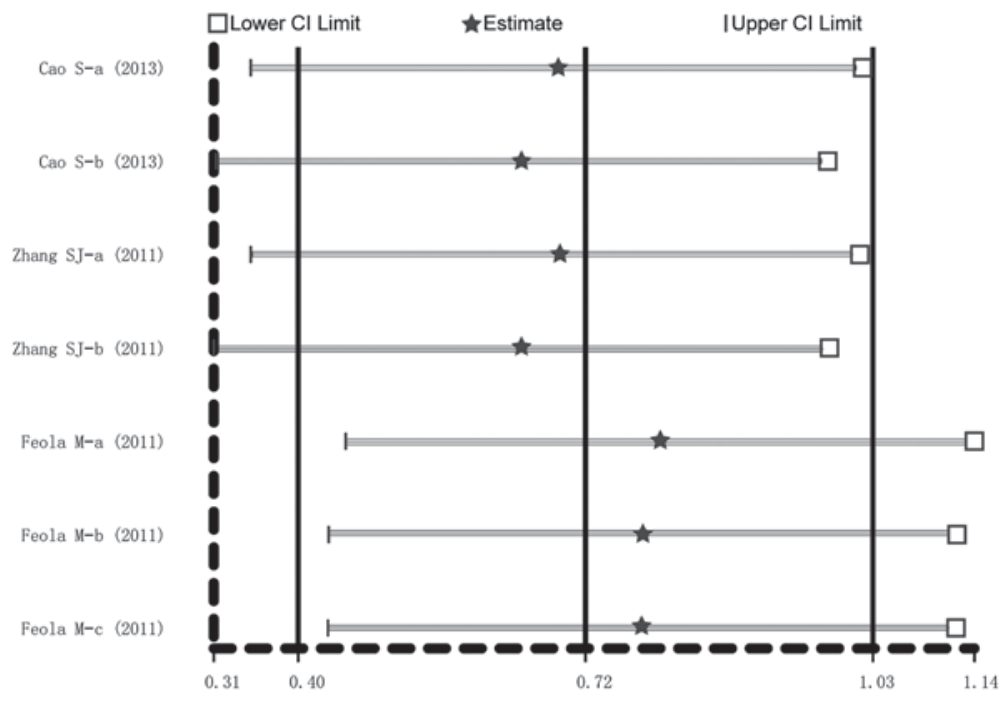

Serum level of BNP in normal (Postreatment vs. Pretreatment)

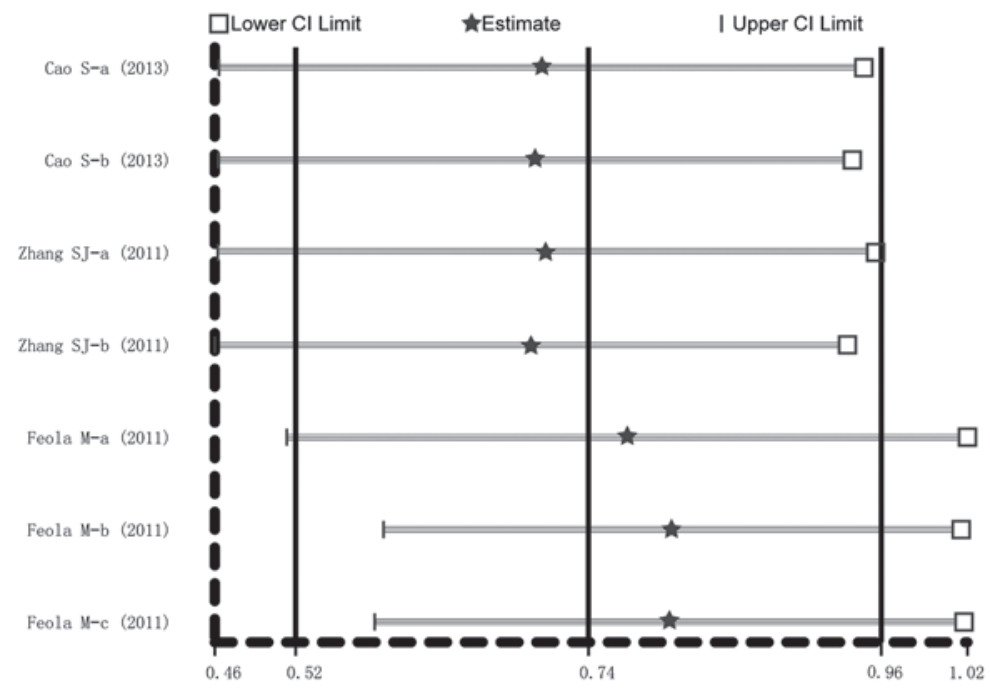

Postreatment serum level of BNP (Cardiotoxic vs. Normal)

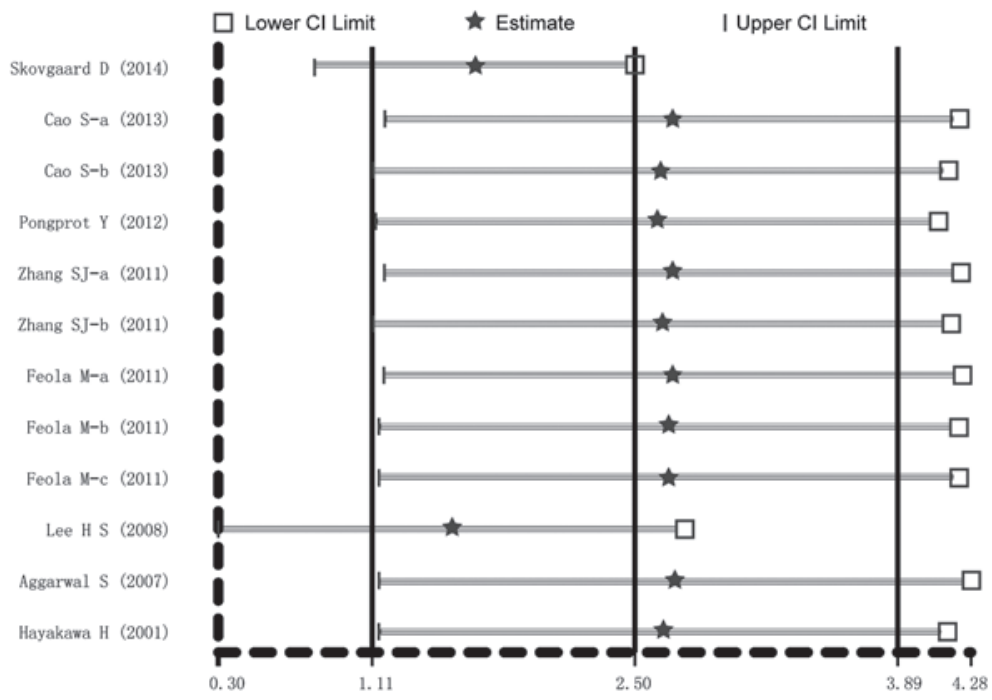

Figure 4. Sensitivity analysis of the summary standardized mean difference on the association between serum B-type natriuretic peptide levels and anthracycline-induced cardiotoxicity. 


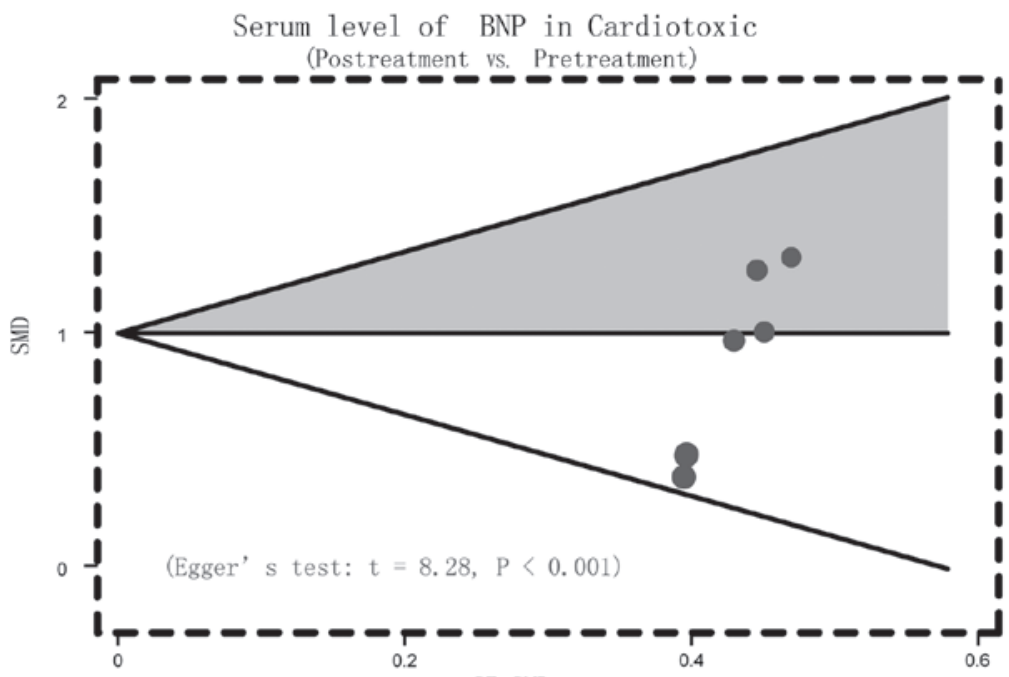

SE SMD

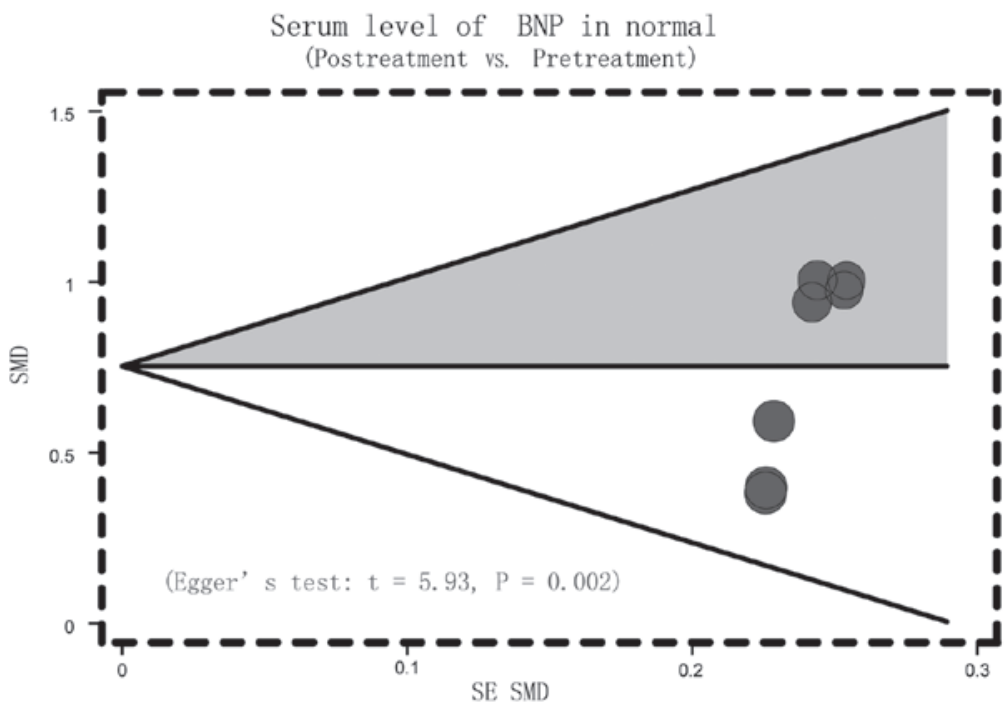

Postreatment serum level of BNP (Cardiotoxic vs. Normal)

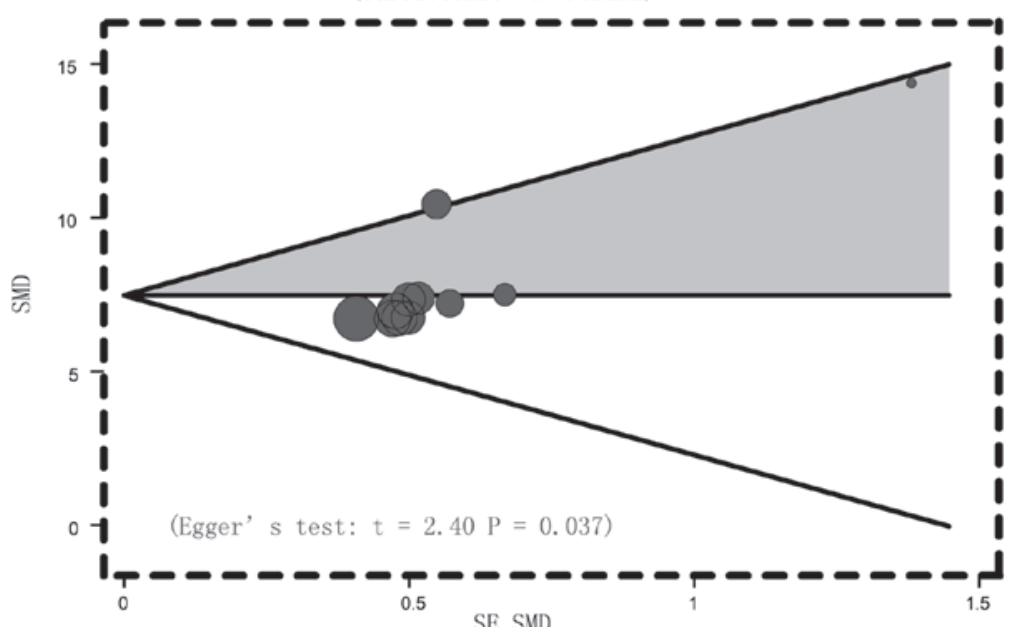

Figure 5. Funnel plot of publication biases in current meta-analysis investigating the association between serum B-type natriuretic peptide levels and anthracycline-induced cardiotoxicity.

serum levels are observed in cardiotoxic patients induced by anthracycline chemotherapy, and BNP serum levels are helpful for predicting, diagnosing and preventing cardiotoxicity. In accordance with the present results, Feola et al (8), supported that the toxic effects following chemotherapy in breast cancer patients may be observed with plasma markers, including BNP, 
to detect left ventricular dysfunction that could provoke cardiac dysfunction (8).

Subgroup analysis was performed in order to take account of other factors that may affect the relationship between BNP serum levels and AIC. The other factors included ethnicity and the detection method. Serum levels of BNP after anthracyclines treatment in cardiotoxic patients were increased among Asian patients, not among Caucasian patients, and the result may be affected by the methods of ECIA and IFT, but not by FIA method, implying that ethnicity and method in the study may be heterogeneity sources. Normal individuals that received anthracycline chemotherapy also exhibited higher serum levels of BNP. BNP detection was obvious among both Asian and Caucasian patients and the three methods of ECIA, IFT and FIA all had the impact on the accuracy of detection. However, when comparing cardiotoxic patients and normal people, increased BNP serum levels were associated with the incidence rates of cardiotoxicity among Caucasian, not among Asian, patients. The association may be influenced by the applied methods like EMIT, ECIA, CIT, IFT, FIA and RIA. Only CLIA method had no effect on the association between BNP levels and cardiotoxicity. The present study revealed that the onset of cardiotoxicity is related with elevated BNP serum levels, and BNP has remarkable clinical value in early diagnosis of AIC patients, improving the overall predictive efficacy in toxic events of the heart.

Limitations in the present study should also be considered. First, there were only 8 articles included, which may not supply sufficient statistical power to estimate the association between the serum levels of BNP and AIC. Second, unpublished studies and articles in languages other than English and Chinese could not be included due to restriction of the inclusion and exclusion criteria, therefore potential heterogeneity and bias may exist. Third, according to the baseline characteristic table, the current meta-analysis only focused on Asian and Caucasian populations, which may also lead to bias. Besides, the sample size and the number of cases were relatively small; the majority were $<20$. In addition, some articles did not contain information about gender, age and follow-up periods. Finally, there may be potential factors that were not taken into account, which may have influenced the results despite all participants of each study were well-defined with similar inclusion criteria, so the results in the current meta-analysis should be interpreted with caution owing to the potential heterogeneity among trials. Despite the described limitations, the current meta-analysis is an important example on the association between the serum levels of BNP and AIC. Of note, a more reliable conclusion was drawn in the meta-analysis, because the inconsistent results were rigorously quantified and analyzed.

In conclusion, the present meta-analysis indicates that the serum level of BNP is associated with AIC. Therefore, serum levels of BNP may be a useful index for monitoring AIC at an early stage. However, further in-depth studies are still required to confirm these findings due to the above limitations.

\section{References}

1. Lee HS, Son CB, Shin SH and Kim YS: Clinical correlation between brain natriutetic peptide and anthracyclin-induced cardiac toxicity. Cancer Res Treat 40: 121-126, 2008.
2. Aapro M, Bernard-Marty C, Brain EG, Batist G, Erdkamp F, Krzemieniecki K, Leonard R, Lluch A, Monfardini S, Ryberg M, et al: Anthracycline cardiotoxicity in the elderly cancer patient: A SIOG expert position paper. Ann Oncol 22: 257-267, 2011.

3. Visscher H, Ross CJ, Rassekh SR, Barhdadi A, Dubé MP, Al-Saloos H, Sandor GS, Caron HN, van Dalen EC, Kremer LC, et al: Pharmacogenomic prediction of anthracycline-induced cardiotoxicity in children. J Clin Oncol 30: 1422-1428, 2012.

4. Lipshultz SE, Alvarez JA and Scully RE: Anthracycline associated cardiotoxicity in survivors of childhood cancer. Heart 94: 525-533, 2008

5. Ewer MS and Lenihan DJ: Left ventricular ejection fraction and cardiotoxicity: Is our ear really to the ground? J Clin Oncol 26: 1201-1203, 2008.

6. van der Pal HJ, van Dalen EC, Hauptmann M, Kok WE, Caron HN, van den Bos C, Oldenburger F, Koning CC, van Leeuwen FE and Kremer LC: Cardiac function in 5-year survivors of childhood cancer: A long-term follow-up study. Arch Intern Med 170: 1247-1255, 2010.

7. Kremer LC, van der Pal HJ, Offringa M, van Dalen EC and Voûte PA: Frequency and risk factors of subclinical cardiotoxicity after anthracycline therapy in children: A systematic review. Ann Oncol 13: 819-829, 2002.

8. Feola M, Garrone O, Occelli M, Francini A, Biggi A, Visconti G, Albrile F, Bobbio M and Merlano M: Cardiotoxicity after anthracycline chemotherapy in breast carcinoma: Effects on left ventricular ejection fraction, troponin I and brain natriuretic peptide. Int J Cardiol 148: 194-198, 2011.

9. Pongprot Y, Sittiwangkul R, Charoenkwan P and Silvilairat S: Use of cardiac markers for monitoring of doxorubixin-induced cardiotoxicity in children with cancer. J Pediatr Hematol Oncol 34: 589-595, 2012.

10. Hayakawa H, Komada Y, Hirayama M, Hori H, Ito M and Sakurai M: Plasma levels of natriuretic peptides in relation to doxorubicin-induced cardiotoxicity and cardiac function in children with cancer. Med Pediatr Oncol 37: 4-9, 2001.

11. Skovgaard D, Hasbak P and Kjaer A: BNP predicts chemotherapy-related cardiotoxicity and death: Comparison with gated equilibrium radionuclide ventriculography. PLoS One 9: e96736, 2014.

12. Beleigoli AM, Boersma E, Diniz Mde F, Vidigal PG, Lima-Costa MF and Ribeiro AL: C-reactive protein and B-type natriuretic peptide yield either a non-significant or a modest incremental value to traditional risk factors in predicting long-term overall mortality in older adults. PLoS One 8: e75809, 2013.

13. Khalaf MA, Abdelrahman TM and Abbas MF: Values of using QTc and N-terminal fragment of B-type natriuretic peptide as markers for early detection of acute antipsychotic drugs-induced cardiotoxicity. Cardiovasc Toxicol 11: 10-17, 2011.

14. Maisel AS, Krishnaswamy P, Nowak RM, McCord J, Hollander JE, Duc P, Omland T, Storrow AB, Abraham WT, Wu AH, et al: Rapid measurement of B-type natriuretic peptide in the emergency diagnosis of heart failure. N Engl J Med 347: 161-167, 2002.

15. Troughton RW, Frampton CM, Yandle TG, Espiner EA, Nicholls MG and Richards AM: Treatment of heart failure guided by plasma aminoterminal brain natriuretic peptide (N-BNP) concentrations. Lancet 355: 1126-1130, 2000.

16. Geske JB, McKie PM, Ommen SR and Sorajja P: B-type natriuretic peptide and survival in hypertrophic cardiomyopathy. $\mathrm{J}$ Am Coll Cardiol 61: 2456-2460, 2013.

17. Gerber IL, Stewart RA, Legget ME, West TM, French RL, Sutton TM, Yandle TG, French JK, Richards AM and White HD: Increased plasma natriuretic peptide levels reflect symptom onset in aortic stenosis. Circulation 107: 1884-1890, 2003.

18. Morrow DA, de Lemos JA, Sabatine MS, Murphy SA, Demopoulos LA, DiBattiste PM, McCabe CH, Gibson CM, Cannon $\mathrm{CP}$ and Braunwald E: Evaluation of B-type natriuretic peptide for risk assessment in unstable angina/non-ST-elevation myocardial infarction: B-type natriuretic peptide and prognosis in TACTICS-TIMI 18. J Am Coll Cardiol 41: 1264-1272, 2003.

19. Berger R, Huelsman M, Strecker K, Bojic A, Moser P, Stanek B and Pacher R: B-type natriuretic peptide predicts sudden death in patients with chronic heart failure. Circulation 105: 2392-2397, 2002.

20. Daniels LB and Maisel AS: Natriuretic peptides. J Am Coll Cardiol 50: 2357-2368, 2007. 
21. Costa VN, Nomura RM, Miyadahira S, Vieira Francisco RP and Zugaib M: Cord blood B-type natriuretic peptide levels in placental insufficiency: Correlation with fetal Doppler and $\mathrm{pH}$ at birth. Eur J Obstet Gynecol Reprod Biol 171: 231-234, 2013.

22. Esteves WA, Lodi-Junqueira L, Neto CP, Tan TC, Nascimento BR, Mehrotra P, Barbosa MM, Ribeiro AL and Nunes MC: The impact of right ventricular stroke work on B-type natriuretic peptide levels in patients with mitral stenosis undergoing percutaneous mitral valvuloplasty. J Interv Cardiol 26: 501-508, 2013.

23. Jan SL, Lin SJ, Fu YC, Lin MC, Chan SC and Hwang B: Plasma B-type natriuretic peptide study in children with severe enterovirus 71 infection: A pilot study. Int J Infect Dis 17: e1166-e1171, 2013.

24. Vila G, Resl M, Stelzeneder D, Struck J, Maier C, Riedl M, Hülsmann M, Pacher R, Luger A and Clodi M: Plasma NT-proBNP increases in response to LPS administration in healthy men. J Appl Physiol (1985) 105: 1741-1745, 2008

25. Clerico A, Giannoni A, Vittorini S and Passino C: Thirty years of the heart as an endocrine organ: Physiological role and clinical utility of cardiac natriuretic hormones. Am J Physiol Heart Circ Physiol 301: H12-H20, 2011.

26. Hequet O, Le QH, Moullet I, Pauli E, Salles G, Espinouse D, Dumontet C, Thieblemont C, Arnaud P, Antal D, et al: Subclinical late cardiomyopathy after doxorubicin therapy for lymphoma in adults. J Clin Oncol 22: 1864-1871, 2004.

27. Stang A: Critical evaluation of the Newcastle-Ottawa scale for the assessment of the quality of nonrandomized studies in meta-analyses. Eur J Epidemiol 25: 603-605, 2010.

28. Zintzaras E and Ioannidis JP: HEGESMA: Genome search meta-analysis and heterogeneity testing. Bioinformatics 21 : 3672-3673, 2005.

29. Zintzaras E and Ioannidis JP: Heterogeneity testing in meta-analysis of genome searches. Genet Epidemiol 28: 123-137, 2005.
30. Higgins JP and Thompson SG: Quantifying heterogeneity in a meta-analysis. Stat Med 21: 1539-1558, 2002 .

31. Song F and Gilbody S: Bias in meta-analysis detected by a simple, graphical test. Increase in studies of publication bias coincided with increasing use of meta-analysis. BMJ 316: 471, 1998.

32. Peters JL, Sutton AJ, Jones DR, Abrams KR and Rushton L: Comparison of two methods to detect publication bias in meta-analysis. JAMA 295: 676-680, 2006.

33. Aggarwal S, Pettersen MD, Bhambhani K, Gurczynski J, Thomas R and L'Ecuyer T: B-type natriuretic peptide as a marker for cardiac dysfunction in anthracycline-treated children. Pediatr Blood Cancer 49: 812-816, 2007.

34. Zhang SJ and Cui YZ: Measurement of brain natriuretic peptide ring based chemotherapy in patients with cardiac toxicity in anthracene. Chinese Journal of Difficult and Complicated Cases 10: 444-445, 2011.

35. Cao S, Pang DM, Duan HB, Lin YD and Li LT: Application value in the levels of plasma NTproBNP in patients with cardiac toxicity. Modern Diagnosis \& Treatment: 303-304, 2013.

36. Mavinkurve-Groothuis AM, Marcus KA, Pourier M, Loonen J, Feuth T, Hoogerbrugge PM, de Korte CL and Kapusta L: Myocardial 2D strain echocardiography and cardiac biomarkers in children during and shortly after anthracycline therapy for acute lymphoblastic leukaemia (ALL): A prospective study. Eur Heart J Cardiovasc Imaging 14: 562-569, 2013.

37. Krawczuk-Rybak M, Dakowicz L, Hryniewicz A, Maksymiuk A, Zelazowska-Rutkowska B and Wysocka J: Cardiac function in survivors of acute lymphoblastic leukaemia and Hodgkin's lymphoma. J Paediatr Child Health 47: 455-459, 2011. 